\title{
Epithelioid Malignant Peripheral Nerve Sheath Tumor (EMPNST) in Children: About A Case and Review of the Literature
}

\author{
Chadi Fadwa $^{1 *}$, Khmou Mouna $^{2}$, Rouas Lamiaa ${ }^{3}$, Lamalmi Najat ${ }^{4}$ \\ ${ }^{1}$ Senior Resident, Department of Pediatric Pathology, Ibn Sina University Hospital, Rabat, Morocco \\ ${ }^{2}$ Practicing Pathologist, Department of Pediatric Pathology, Ibn Sina University Hospital, Rabat, Morocco \\ ${ }^{3}$ Senior Professor, Department of Pediatric Pathology, Ibn Sina University Hospital, Rabat, Morocco \\ ${ }^{4}$ Senior Professor and Head, Department of Pediatric Pathology, Ibn Sina University Hospital, Rabat, Morocco
}

\author{
DOI: $\underline{10.36348 / \text { sipm.2020.v05i12.010 }}$ \\ | Received: 13.12.2020 | Accepted: 24.12.2020 | Published: 30.12.2020 \\ *Corresponding author: Chadi Fadwa
}

\section{Abstract}

Epithelioid malignant peripheral nerve sheath tumor (EMPNST) known as "epithelioid malignant schwannoma", described for the first time in 1954. It is an entity that has the same clinical features as MPNST but whose cytological and histological characters are different. It is characterized by an epithelioid cytomorphology, usually diffuse S100 immunoreactivity and some cases come from preexisting schwannomas. Clinically, it presents as a painful or asymptomatic mass, whether or not it is located on a nerve path. Imaging plays an essential role in preoperative diagnosis and assessment, but histology remains the key examination for confirmation of the diagnosis. Treatment is based on surgery, radiation therapy and chemotherapy. His prognosis is grim. The objective of this work is to underline the histopathological aspects of this very rare entity, in particular in children, by reporting an observation of a 21 month old infant.

Keywords: Epithelioid malignant peripheral nerve sheath tumor, Malignant peripheral nerve sheath tumor, child, infant, histopathology.

Copyright (C) 2020 The Author(s): This is an open-access article distributed under the terms of the Creative Commons Attribution 4.0 International License (CC BY-NC 4.0) which permits unrestricted use, distribution, and reproduction in any medium for non-commercial use provided the original author and source are credited.

\section{INTRODUCTION}

Peripheral nerve sheath malignancies (MPNST) represent 5\% of soft tissue malignancies and approximately $10 \%$ of soft tissue sarcomas. They are difficult to diagnose given their great anatomo pathological heterogeneity. The epithelioid type represents a variant constituting less than $5 \%$ of MPNST which characterizes by the predominance of epithelioid cells, diffuse S100 positivity and infrequent association with neurofibromatosis type 1 . The purpose of this work is to highlight the histopathological aspects of this very rare entity, in particular in children by reporting an observation of a 21 month old infant.

\section{CASE REPORT}

This is for a female child of 21-month with localized swelling in the elbow crease level and without remote extension, having relapsed one month after a first resection. X-ray of the face and profile of the elbow shows swelling of the soft tissues. Ultrasound of the elbow reveals a heterogeneous oblong, bilobed and circumscribed tissue process measuring $35 \times 17 \mathrm{~mm}$. CT of the sagittal elbow shows an irregular mass of muscle density. The MRI revealed a hypointense process T1 and hyperintense T2 with contrast enhancement, infiltrating the muscles of the anterior compartment of the upper third of the forearm, the biceps tendon and the brachial subcutaneous fat with epitrochlear lymphadenopathy.

Macroscopic examination shows a tumor measuring $4 \mathrm{~cm}$ long, firm and whitish, sheathing a nerve thread.

The microscopic examination focused on a proliferation of spindle-shaped and epithelioid cells with a round nucleolus nucleus and pseudo-glandular lobulated architecture with tumor necrosis estimated at $20 \%$. An infiltration of connective-muscular tissue and nerve fibers was noted.

The immunostaining was frank for PS100, vimentin, cytokeratin AE1/AE3 and CAM5.2, focally positive for CD99 and AML and absent for EMA, HMB45, TLE1, Desmin and Myogenin. The diagnosis retained was an epithelioid-type malignant nerve sheath tumor (EMPNST) of intermediate risk, classified grade II by the system of the Children Oncology Group 
(COG) and the National Federation of Centers for the Fight against Cancer (FNCLCC)

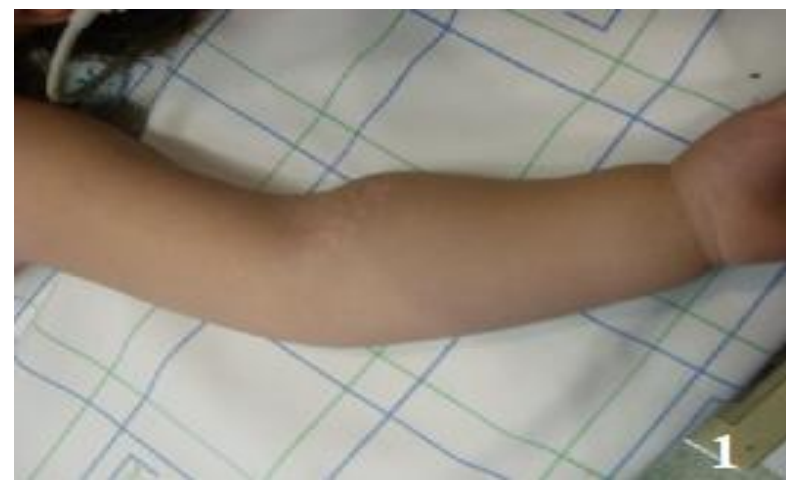

Fig-1: Recurrence of MPNSTs at the elbow with scar from the $1^{\text {st }}$ operation

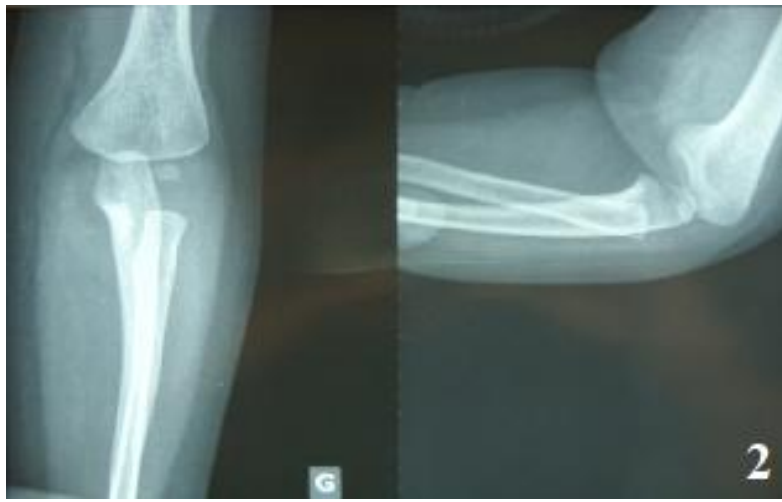

Fig-2: Elbow X-ray face and profile showing a soft tissue swelling

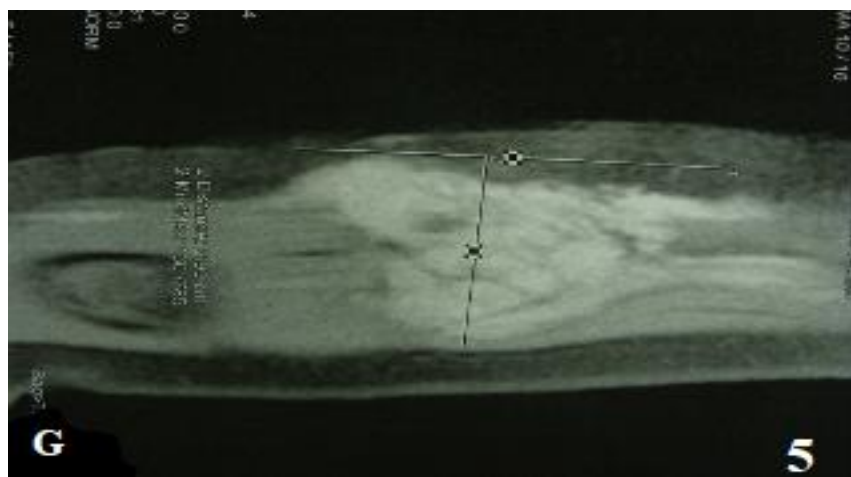

Fig 5 \& 6: Elbow MRI (sagittal (5) and transverse (6)): A process that takes up contrast and infiltrates the muscles of the anterior compartment of the forearm

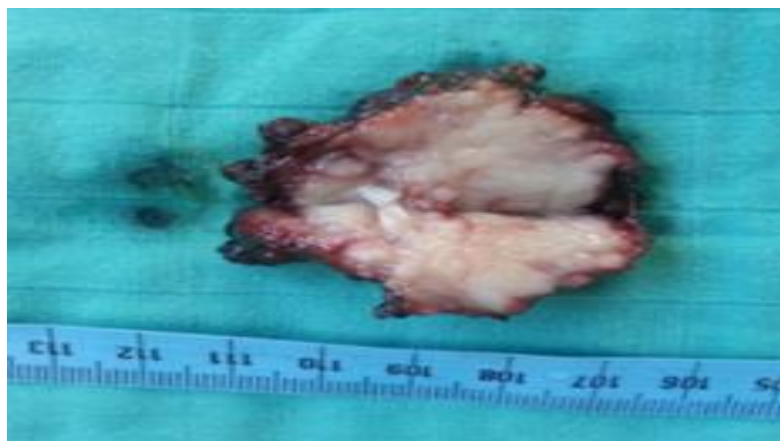

Fig-7: Tumor measuring 4cm long, firm, whitish and sheathing a nervous thread
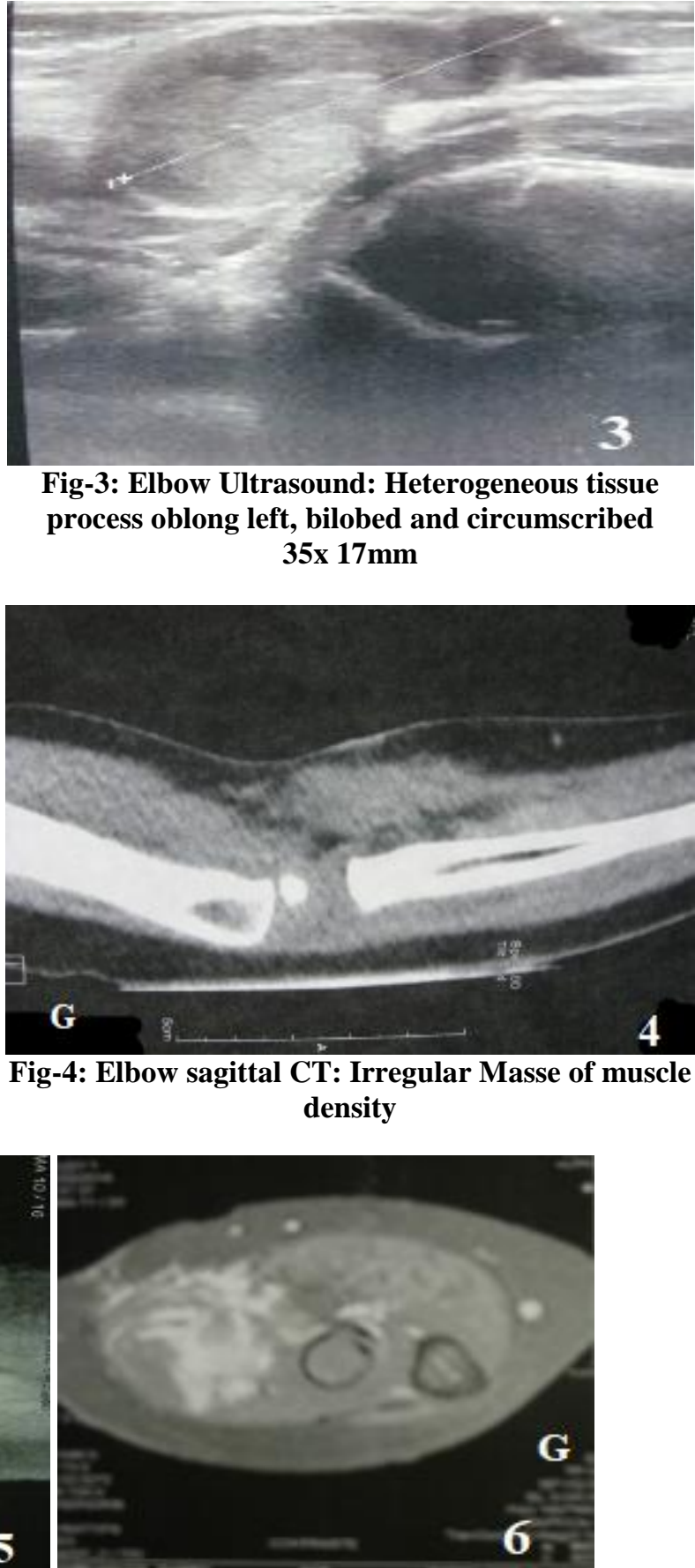

Fig-3: Elbow Ultrasound: Heterogeneous tissue process oblong left, bilobed and circumscribed $35 \times 17 \mathrm{~mm}$

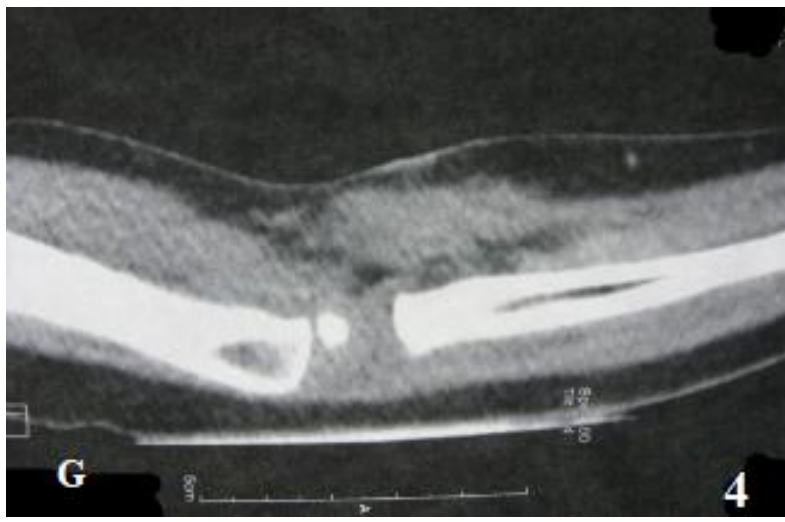

Fig-4: Elbow sagittal CT: Irregular Masse of muscle density

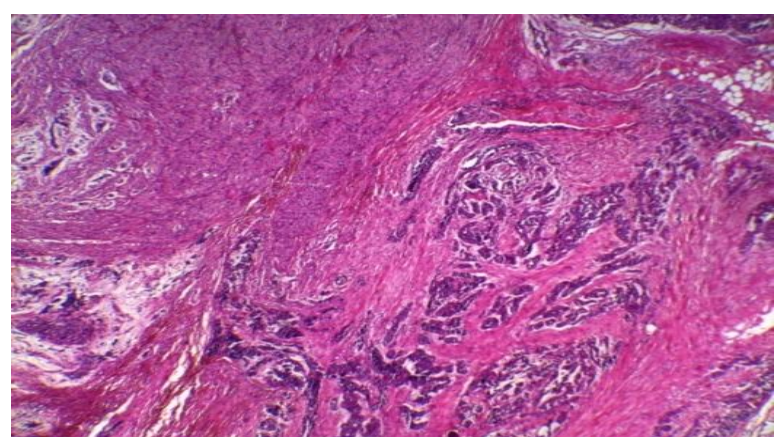

Fig-8: Nests and rows of cells infiltrating a large nerve thread at the top and left and fatty tissue on the right

(HEx4) 


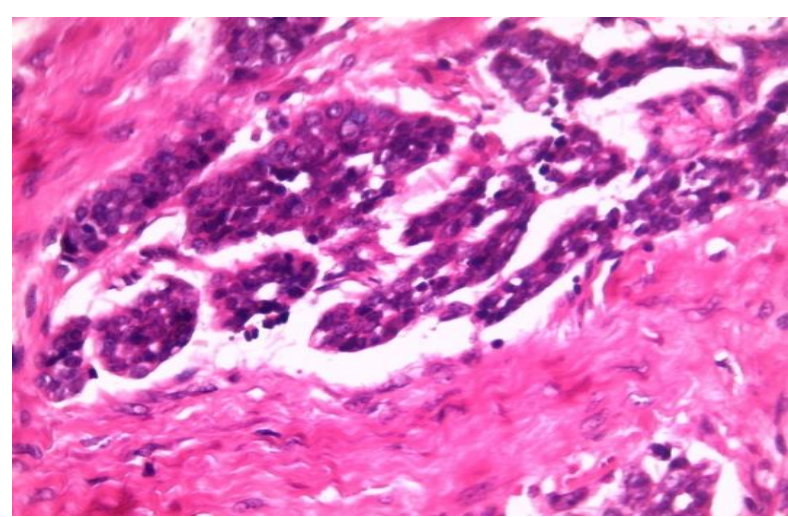

Fig-9: Pseudo-glandular appearance (HEx40)

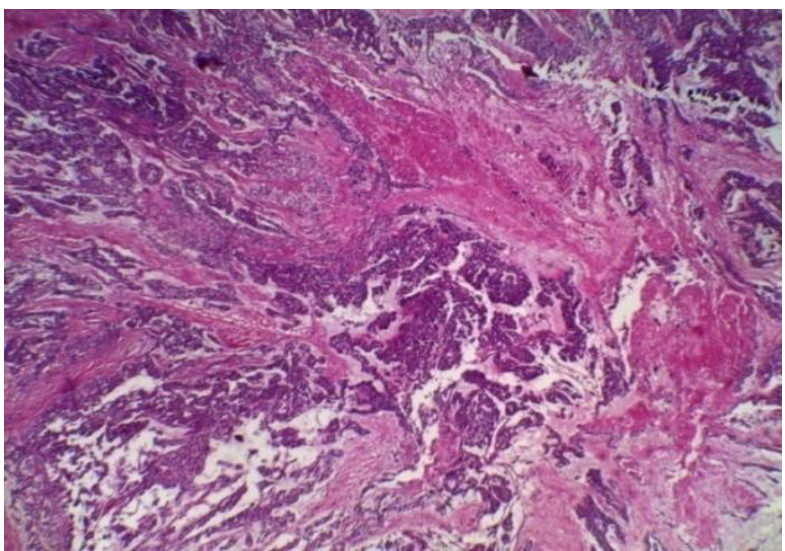

Fig-10: Central tumor necrosis (HEx40)

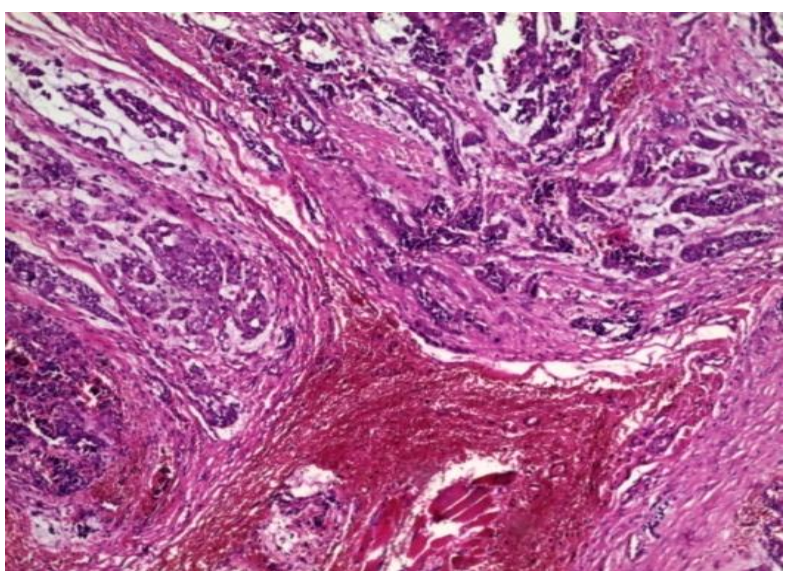

Fig-11: Infiltration of striated muscle tissue (HEx40)

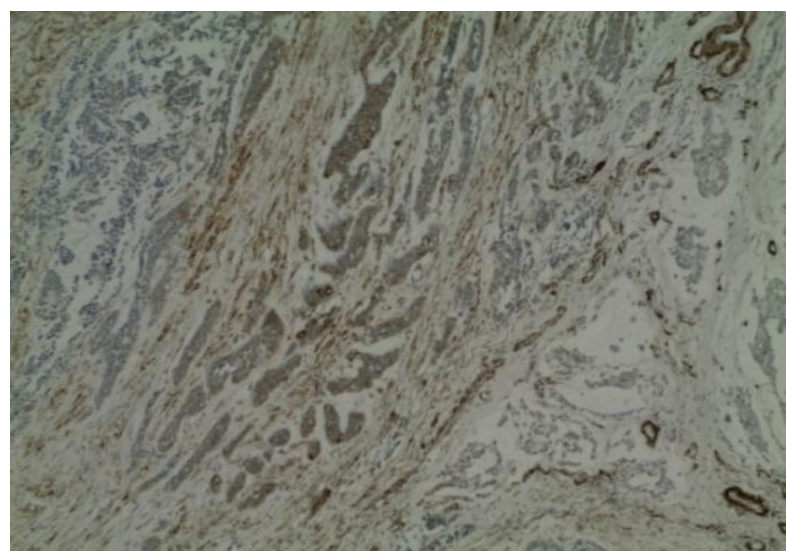

Fig-12: Anti-S100 + antibody

\section{DISCUSSION}

Epithelioid malignant peripheral nerve sheath tumor (EMPNST), is a particular variant of malignant peripheral nerve sheath tumor, accounting for less than $5 \%$ of these tumors [1], characterized by the predominance of epithelioid cells [2]. She has been described for the first time in 1954 [3]; There have been few documented case series on this rare entity. Lately, Yo and Fletcher described the clinicopathologic spectrum of a fairly large series of EMPNST [2]. These are usually tumors of young adults, between the ages of 20 and 50. Overall, they occur more in cases of associated neurofibromatosis (28-36 years) than outside this context (40-44 years). Cases have been reported in children and adolescents, as well as in the elderly. There is no gender predominance [4]. Development of an EMPNST is associated with malignant epitheloid change in a schwannoma, as elucidated by previous authors, in their seminal articles [1]. These are rare, ubiquitous, often painful tumors, usually but developing irregularly along the path of a nerve, with a poor prognosis [4]. While a previous study suggested a more favorable clinical course for EMPNST in superficial compared with deep location, the largest series of EMPNST to date showed a comparatively low risk of recurrence and metastasis, irrespective of tumor depth. Pain is the most frequent clinical sign: neuralgia or indefinable pain; but peripheral tumors can be asymptomatic. On examination, the lesions present in the form of a large mass, evolving several months before diagnosis [4]. Imaging is essential today for the diagnostic approach and the preoperative assessment of these tumors. Magnetic resonance imaging (MRI) plays a key role, combined with computed tomography [4]. Histologically, EMPNST displays a multilobulated growth pattern and is characterized by epithelioid cells with vesicular nuclei, prominent nucleoli, and amphophilic or eosinophilic cytoplasm, sometimes in a chondromyxoid stroma. Marked cytologic atypia is evident in most cases. Chondroosseous differentiation is rare, with no other mesenchymal (eg., Rhabdomyoblastic) elements reported so far [3]. The cytological study of the epithelioid variant shows an abundant cytoplasm, with adhesive tendency of the cells and appearance of plasmocytoid or epithelioid characters, which is not the case with other MPNST where there is a spindle appearance [4].

On the immunohistochemical, EMPNST shows a strong expression of diffuse S100 in almost all cases, as well as GFAP in $60 \%$ and EMA in $15 \%$ of cases. The trimethylation of a histone H3K27 (H3K27me3) is preserved in all cases of EMPNST. $67 \%$ of EMPNST show a loss of expression of SMARCB1 (INI1) [3]. The antibodies anti-cytokeratin negative, as are anti-HMB [4]. These immunohistochemical characteristics allow a differential diagnosis of benign nerve tumors, other sarcomas and melanomas. To further support the diagnosis, other means of study could be used, such as 
molecular biology and cytogenetics [4]. The various differential diagnoses considered were an epithelioid schwannom, a melanoma, metastatic carcinoma, epithelioid sarcoma, myoepithelial tumors, and extraosseous myxoid chondrosarcoma [1]. Metastases are hematogenous or follow the path of the nerve sheaths. Distant metastases are localized in the lungs, liver and bones. They appear within an average of two years, which is even shorter when there is type 1 neurofibromatosis [4].

EMPNST can be treated by a combination of surgery, radiotherapy and chemotherapy [3]. MPNSTs, including EMPNST are moderately chemosensitive tumors. Recently, there has been an isolate report of vemurafenib treatment of BRAF V600E mutated malignant peripheral nerve sheath tumor [1]

\section{CONCLUSION}

To conclude, EMPNSTs are very rare tumors especially in children, with a poor prognosis. They represent a polymorphic histopathological aspect, characterized by a multilobulated appearance tumor with a predominance of epithelioid cells and a diffuse positivity of the S100 protein in most cases. Although imaging has an interest in diagnosis, pathological anatomy remains the key element in confirming the diagnosis. Most tumors are treated by a surgical resection.

\section{REFERENCES}

1. Rekhi, B., Kosemehmetoglu, K., Tezel, G. G., \& Dervisoglu, S. (2017). Clinicopathologic features and immunohistochemical spectrum of 11 cases of epithelioid malignant peripheral nerve sheath tumors, including INI1/SMARCB1 results and BRAF V600E analysis. Apmis, 125(8), 679-689.

2. Luzar, B., Shanesmith, R., Ramakrishnan, R., Fisher, C., \& Calonje, E. (2016). Cutaneous epithelioid malignant peripheral nerve sheath tumour: a clinicopathological analysis of 11 cases. Histopathology, 68(2), 286-296.

3. Hung, Y. P., \& Hornick, J. L. (2017). Epithelioid soft tissue tumors: a clinicopathologic and molecular update. AJSP: Reviews \& Reports, 22(2), 62-74.

4. Soualhi, M., El Ouazani, H., Chaibainou, A., Bouchentouf, R., El Ftouh, M., Mouline, S., \& Fihry, M. E. F. (2004). Tumeur maligne des gaines des nerfs périphériques type épithélioïde: A propos d'un cas. Revue de Pneumologie Clinique, 60(1), 50-54. 